\title{
Heterogeneous Kinetics and Pharmacology of Synaptic Inhibition in the Chick Auditory Brainstem
}

\author{
Sidney P. Kuo, Laura A. Bradley, and Laurence 0. Trussell \\ Oregon Hearing Research Center and Vollum Institute, Oregon Health and Science University, Portland, Oregon 97239
}

Identification of shared features between avian and mammalian auditory brainstem circuits has provided much insight into the mechanisms underlying early auditory processing. However, previous studies have highlighted an apparent difference in inhibitory systems; synaptic inhibition is thought to be slow and GABAergic in birds but to have fast kinetics and be predominantly glycinergic in mammals. Using patch-clamp recordings in chick brainstem slices, we found that this distinction is not exclusively true. Consistent with previous work, IPSCs in nucleus magnocellularis (NM) were slow and mediated by $\mathrm{GABA}_{\mathrm{A}}$ receptors. However, IPSCs in nucleus laminaris (NL) and a subset of neurons in nucleus angularis (NA) had rapid time courses twofold to threefold faster than those in NM. Furthermore, we found that IPSCs in NA were mediated by both glycine and $\mathrm{GABA}_{\mathrm{A}}$ receptors, demonstrating for the first time a role for fast glycinergic transmission in the avian auditory brainstem. Although NM, NL, and NA have unique roles in auditory processing, the majority of inhibitory input to each nucleus arises from the same source, ipsilateral superior olivary nucleus (SON). Our results demonstrate remarkable diversity of inhibitory transmission among the avian brainstem nuclei and suggest that differential glycine and $\mathrm{GABA}_{\mathrm{A}}$ receptor activity tailors inhibition to the specific functional roles of NM, NL, and NA despite common SON input. We additionally observed that glycinergic/GABAergic activity in NA was usually depolarizing and could elicit spiking activity in NA neurons. Because NA projects to SON, these excitatory effects may influence the recruitment of inhibitory activity in the brainstem nuclei.

\section{Introduction}

The vertebrate auditory system uses several environmental cues to determine the location of sound sources, including the relative timing and intensity of acoustic signals between the two ears (Konishi, 2003). In both birds and mammals, specialized circuits devoted to these distinct sound characteristics are established in the brainstem. In birds, auditory nerve fibers diverge to innervate nucleus magnocellularis (NM) and nucleus angularis (NA) (Parks and Rubel, 1978). Neurons in NM bilaterally relay precise timing information to targets in nucleus laminaris (Boord, 1968; Parks and Rubel, 1975; Fukui et al., 2006). Nucleus laminaris (NL) neurons rely on these signals to compute submillisecond differences in sound arrival time between each ear that arise when a sound originates closer to one side of the head, termed interaural timing differences (ITDs) (Carr and Konishi, 1990; Konishi, 2003). In contrast, NA is thought to represent the first stage in the processing of sound intensity, as well as other nontiming aspects of auditory signals (Sullivan and Konishi, 1984; Takahashi et al., 1984; MacLeod and Carr, 2007).

Examination of the intrinsic properties of auditory brainstem neurons as well as their excitatory inputs have revealed similar

Received Jan. 7, 2009; revised June 9, 2009; accepted June 24, 2009.

This work was supported by National Institutes of Health Grant R01 DC004450 (L.O.T.) and a Cornelia H. Stevens Achievement Rewards for College Scientists scholarship (S.P.K.). We thank K. Bender, Y. Kim, C. Mello, K. Spinelli, T. Velho, and H. Zhao for advice and technical assistance, M. Roberts for providing Igor analysis procedures and advice, and P. Gillespie and Gillespie laboratory members for sharing equipment.

Correspondence should be addressed to Sidney P. Kuo, Oregon Health and Science University, 3181 SW Sam Jackson Park Road, L335A, Portland, OR 97239. E-mail: kuos@ohsu.edu.

DOI:10.1523/JNEUROSCI.0103-09.2009

Copyright (C) 2009 Society for Neuroscience $\quad 0270-6474 / 09 / 299625-10 \$ 15.00 / 0$ anatomical and biophysical specializations in the avian and mammalian brainstem nuclei, indicating shared principles for encoding sound (Oertel, 1999; Trussell, 1999; Carr and Soares, 2002). However, investigations of inhibitory synaptic transmission suggested a striking distinction between birds and mammals. In mammals, inhibition undergoes a developmental switch from GABAergic in immature animals to predominantly glycinergic transmission in the adult (Kotak et al., 1998; Smith et al., 2000; Nabekura et al., 2004; Awatramani et al., 2005). Glycinergic currents in the mammalian brainstem typically have rapid kinetics, with decay time constants of only a few milliseconds or less (Smith et al., 2000; Awatramani et al., 2004; Magnusson et al., 2005), and can retain the phase-locked temporal structure of auditory signals. In contrast, inhibition in the avian auditory brainstem is thought to be GABAergic throughout development (Code and Rubel, 1989; von Bartheld et al., 1989; Lachica et al., 1994). In NM, in which IPSCs have slow kinetics, GABA release becomes asynchronous at high rates of activity and IPSCs summate to mediate tonic inhibition ( $\mathrm{Lu}$ and Trussell, 2000). The anatomy of inhibition is also unique in birds; inhibition to NM, $\mathrm{NL}$, and NA arises predominantly from the same source, ipsilateral superior olivary nucleus (SON) (Lachica et al., 1994; Monsivais et al., 2000; Burger et al., 2005), which is not thought to provide phase-locked inhibition to its targets (Lachica et al., 1994; Yang et al., 1999). SON receives excitatory projections from ipsilateral NL and NA. These distinctions have led to the suggestion that inhibition serves fundamentally different roles in birds and mammals (Grothe, 2003). We tested this generalization by examining inhibitory transmission in NM, NL, and NA neurons in slices of embryonic chick brainstem. 
Table 1. Summary of IPSC properties in chick auditory brainstem

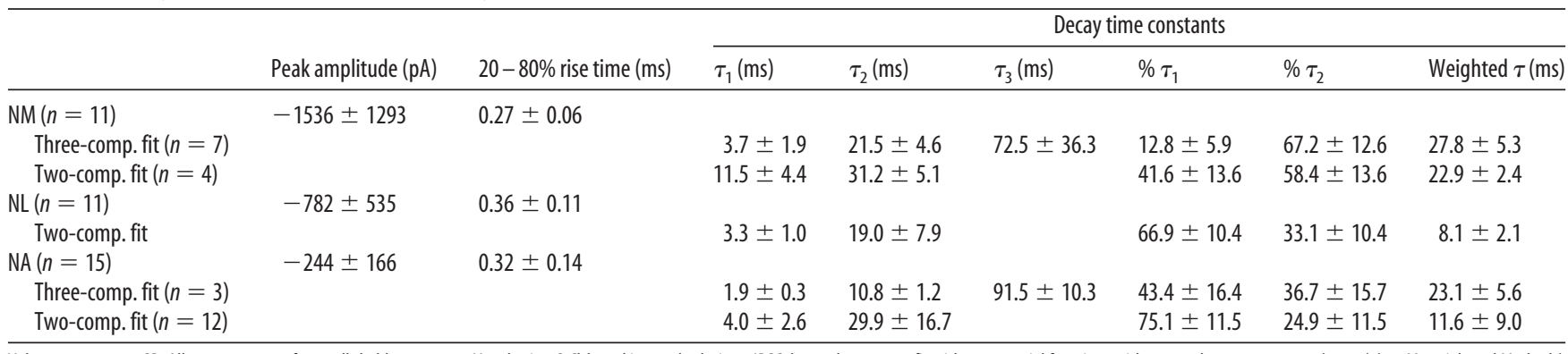

Values are mean \pm SD. All measurements from cells held at $-35.9 \mathrm{mV}$ and using (sCl-based internal solutions. IPSC decay phases were fit with exponential functions with two or three components (comp.) (see Materials and Methods).

\section{Materials and Methods}

Tissue preparation. Coronal slices of brainstem (250 $\mu \mathrm{m}$ thick) were prepared from 17- to 20-d-old chick embryos [embryonic day 17 (E17) to E20]. In experiments using hatchling chicks (post-hatch day 0), chicks were deeply anesthetized with isoflurane before they were killed. After decapitation, a portion of brainstem containing the auditory nuclei was blocked in the coronal plane and affixed to the stage of a vibratome (Leica VT1000S or VT1200S) and then sectioned. During dissection and slicing, tissue remained immersed in an oxygenated saline solution containing the following (in mM): $140 \mathrm{NaCl}, 5 \mathrm{KCl}, 3 \mathrm{CaCl}_{2}, 1 \mathrm{MgCl}_{2}, 10 \mathrm{HEPES}$, and 10 glucose, $\mathrm{pH} 7.4$ using $\mathrm{NaOH}$ (warmed to $\left.\sim 35^{\circ} \mathrm{C}\right)(300 \mathrm{mOsm})$. After sectioning, slices were allowed to recover in the same solution at $35^{\circ} \mathrm{C}$ for $1 \mathrm{~h}$ and then transferred to a recording chamber or maintained at room temperature $\left(\sim 22^{\circ} \mathrm{C}\right)$ until use.

Electrophysiology. During recordings, slices were continuously perfused with oxygenated saline solution identical to that used for slice preparation, but with the addition of 6,7-dinitroquinoxaline-2,3-dione (DNQX) $(20 \mu \mathrm{M})$ and DL-2-amino-5-phosphonopentanoic acid (DLAPV) $(100 \mu \mathrm{M})$. For all experiments, bath solution was warmed to $35 \pm$ $1^{\circ} \mathrm{C}$ using an in-line heater (Warner Instruments ). Bath temperature was constantly monitored with a thermister positioned at the tip of the microscope objective. Cells were visualized for recordings on the stage of an upright microscope (Olympus BX51W) using infrared Dodt gradient contrast optics and a $60 \times$ magnification objective. Neurons in NM, NL, and NA were identified by cellular morphology and location within the slice. Recordings from NM and NL were obtained from the caudal onethird of the nuclei, corresponding to the middle to low characteristic frequency regions (Rubel and Parks, 1975). In this study, we did not explore whether tonotopic variation in IPSC properties exists in NM and NL. In NA, cells were recorded throughout the extent of the nucleus. For whole-cell voltage-clamp experiments, patch pipettes were filled with one of two CsCl-based internal solutions. One contained the following (in $\mathrm{mm}$ ): $130 \mathrm{CsCl}, 2 \mathrm{MgCl}_{2}, 10$ tetraethylammonium (TEA)-Cl, 10 HEPES, 4 BAPTA-Cs $s_{4}$, and $4 \mathrm{Mg}$-ATP, pH 7.2 with $\mathrm{CsOH}(290 \mathrm{mOsm})$. The other consisted of the following (in $\mathrm{mM}$ ): $120 \mathrm{CsCl}, 1.4 \mathrm{MgCl}_{2}, 10$ TEA-Cl, $0.4 \mathrm{Na}_{2}$-GTP, 10 Tris $_{2}$-phosphocreatine, $4 \mathrm{Mg}$-ATP, $10 \mathrm{HEPES}$, and 4 BAPTA-Cs $_{4}$, pH 7.2 with $\mathrm{CsOH}(290$ mOsm). QX-314 [N-(2,6dimethylphenylcarbamoylmethyl)triethylammonium chloride] (1 $\mathrm{mM})$ was sometimes included in the internal solution. IPSCs recorded with either internal solution and with or without QX-314 did not exhibit different decay kinetics or pharmacology, so data using the different $\mathrm{CsCl}$-based internal solutions were pooled. To confirm that strychnine blocked a $\mathrm{Cl}^{-}$-mediated current in NA neurons (see Results), some recordings were obtained using a low $\left[\mathrm{Cl}^{-}\right]$internal solution containing the following (in mM): $108 \mathrm{CsMeSO}_{3}, 5 \mathrm{CsCl}, 1.4 \mathrm{MgCl}_{2}, 0.4 \mathrm{Na}$-GTP, 15 Tris $_{2}$-phosphocreatine, 4 Mg-ATP, 10 HEPES, and 8 BATPA-Cs $_{4}$, pH 7.2 with $\mathrm{CsOH}(290 \mathrm{mOsm})$. These measurements were not included in the reported averages. To assess the action potential firing phenotype of NA neurons, recordings from a subset of NA cells were acquired using a $\mathrm{K}^{+}$-based internal solution containing the following (in $\mathrm{mM}$ ): 81 K-gluconate, $32 \mathrm{KCl}, 4.5 \mathrm{MgCl}_{2}$, 14 Tris 2 -phosphocreatine, $4 \mathrm{Na}_{2}$-ATP, 0.3 Tris-GTP, 9 HEPES, 0.1 EGTA, and 0.2 sucrose, pH 7.2 with $\mathrm{KOH}$ (290 mOsm). All recording pipettes were pulled from borosilicate glass (WPI) and had a resistance of 1.5-3 M $\Omega$ when filled with CsCl-based internal solution. Reported membrane potential values were corrected for empirically determined junction potentials of -5.9 and $-11.4 \mathrm{mV}$ for CsCl-based and $\mathrm{K}^{+}$-based internal solutions, respectively. IPSCs were evoked by locally applying electrical stimuli $(<50 \mu \mathrm{m}$ from soma; $20-60 \mathrm{~V}, 100-200 \mu \mathrm{s})$ through double-barreled glass pipettes $(5-10 \mu \mathrm{m}$ tip diameter) filled with bath solution. In experiments examining evoked IPSCs using the CsCl-based internal solutions, the membrane potential of the recorded cell was held at $-35.9 \mathrm{mV}$. Evoked IPSCs were recorded at $-71.4 \mathrm{mV}$ in experiments using the $\mathrm{K}^{+}$-based pipette solution. Spontaneously occurring IPSCs (sIPSCs) were measured while the membrane potential of the recorded cell was maintained at $-65.9 \mathrm{mV}$. For gramicidin perforated-patch recordings, pipettes were filled with a solution containing the following: $140 \mathrm{~mm} \mathrm{KCl,} 10 \mathrm{~mm} \mathrm{NaCl}, 10 \mathrm{~mm}$ HEPES, 60-100 $\mu \mathrm{g}$ of gramicidin D, and $50 \mu \mathrm{m}$ Alexa Fluor 488. After seal formation, series resistance $\left(R_{\mathrm{s}}\right)$ was monitored in voltage-clamp mode by applying a $10 \mathrm{mV}$ voltage step across the pipette tip. Experiments were initiated once $R_{\mathrm{s}}$ declined to $20-50 \mathrm{M} \Omega$, which typically took $20-30 \mathrm{~min}$. The integrity of the membrane under the recording electrode was confirmed periodically by checking that Alexa Fluor 488 signal was excluded from the recording pipette. Gramicidin perforated-patch measurements were not corrected for junction potential (Kim and Trussell, 2007). Glycine (1 $\mathrm{mm}$ in bath solution) was pressure-applied using a picospritzer $(100 \mathrm{~ms}$, $\sim 1-2$ psi application; $1 \mathrm{psi}=6.89 \mathrm{kPa}$; Parker Instrumentation) through a patch pipette positioned adjacent to the soma of the recorded cell. SR-95531 [6-imino-3-(4-methoxyphenyl)-(6H)-pyridazinebutanoic acid hydrobromide] and strychnine were applied by bath perfusion. Pharmacological agents were obtained from Ascent Scientific except for strychnine, which was from Sigma-Aldrich. Extracellular and intracellular solution components were obtained from Sigma with the following exceptions: BAPTA-Cs $s_{4}$ and Alexa Fluor 488 were from Invitrogen, and QX-314 was from Alomone Labs.

Electrophysiological data acquisition and analysis. Recordings were acquired using a MultiClamp 700B amplifier (Molecular Devices) and pClamp 10.0 software. Signals were digitized at 20 or $50 \mathrm{kHz}$ using a Digidata 1322A (Molecular Devices) and low-pass filtered at $10 \mathrm{kHz}$. For whole-cell voltage-clamp experiments, series resistance $(<15 \mathrm{M} \Omega)$ was compensated $60-80 \%$ during recordings. Series resistance was left uncompensated during perforated-patch recordings and was instead monitored continuously throughout the experiments by measuring the peak of the instantaneous current response to $5-10 \mathrm{mV}$ voltage step commands. Membrane potential values for perforated-patch recordings were corrected offline by subtracting the voltage error, calculated by multiplying holding current by series resistance, from the command holding potential. Analyses were conducted using Clampfit 10.0 (Molecular Devices) or custom procedures written in IgorPro (WaveMetrics) (procedures written by M. T. Roberts, University of Texas, Austin, TX). The decay phases of evoked IPSCs were fit with exponential functions with one, two, or three components. The best fit was selected by comparing the sum of squared errors between fits with different numbers of exponential components. We selected those fits that resulted in a sum of squared errors of less than half that of a fit with fewer components. Spontaneous IPSCs were detected and aligned using the variableamplitude template function event detection feature of Axograph X. Because of the small amplitudes of many of the spontaneous IPSCs, we 
A

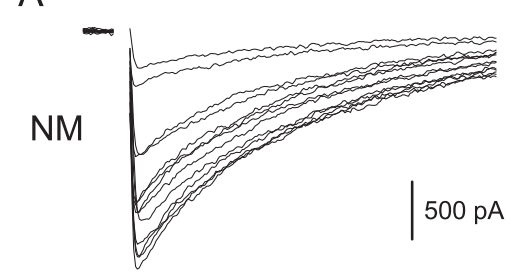

C

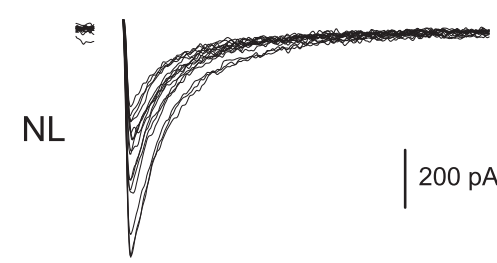

E

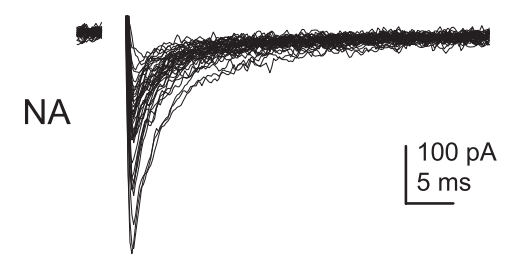

B

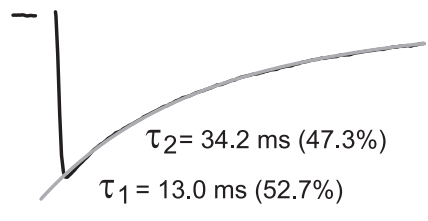

D

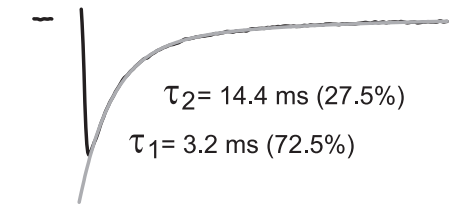

$\mathrm{F}$

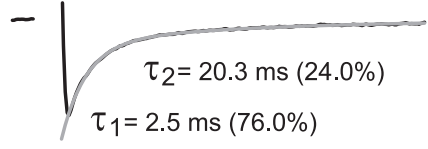

Figure 1. Divergent time courses of inhibition in chick auditory brainstem nuclei. Example stimulus-evoked IPSC recordings from neurons in nucleus magnocellularis $(\boldsymbol{A}, \boldsymbol{B})$, nucleus laminaris $(\boldsymbol{C}, \boldsymbol{D})$, and nucleus angularis $(\boldsymbol{E}, \boldsymbol{F}) . \boldsymbol{A}, \boldsymbol{C}, \boldsymbol{E}$, Individual, overlaid current traces from stimuli applied at 0.2 or $0.5 \mathrm{~Hz}$. $\boldsymbol{B}, \boldsymbol{D}, \boldsymbol{F}$, Averaged current traces (10-20 stimuli). Gray lines are biexponential fits to the current decay phases. Stimulus artifacts in this and subsequent figures have been blanked for clarity.

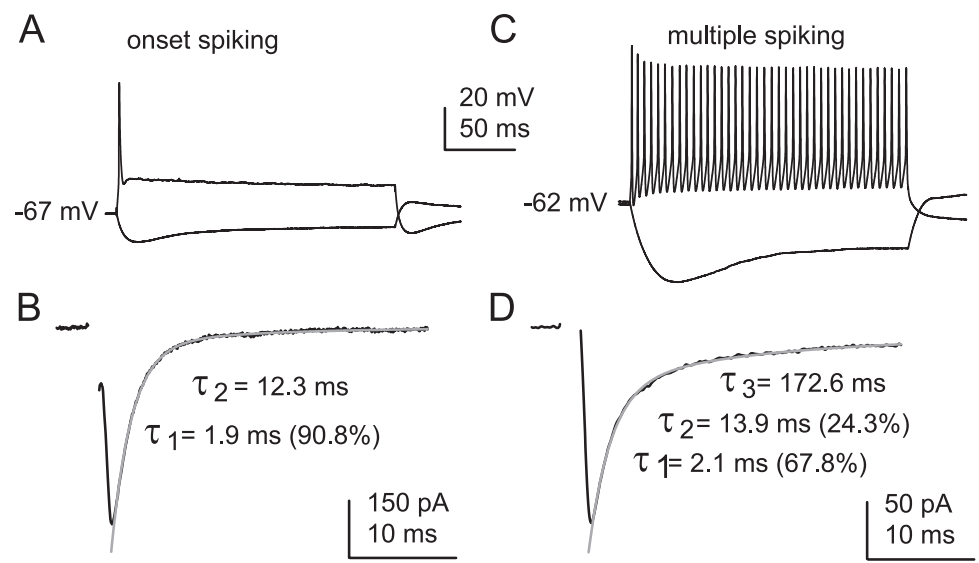

Figure 2. Unique IPSC decay kinetics between onset spiking and multiple spiking neurons in NA. $\boldsymbol{A}$, Response to -200 and +600 pA current injection in an example onset spiking neuron. $\boldsymbol{B}$, Average evoked IPSC in same cell as in $\boldsymbol{A}$. C, Multiple spiking neuron response to -200 and +600 pA injection. $\boldsymbol{D}$, Evoked IPSC measured from same cell shown in $\boldsymbol{C}$. Gray lines in $\boldsymbol{B}$ and $\boldsymbol{D}$ show exponential fit to IPSC decay phase.

determined their time courses by normalizing all events to their peak amplitudes and fitting a single-exponential function to decay phases of the IPSCs. Fits obtained in this manner were visually inspected, and events in which we were unable to satisfactorily fit the data, which represented a small minority of the total number of events, were removed. All reported values are mean \pm SD. Unless noted otherwise, statistical significance $(p<0.05)$ was tested using unpaired two-tailed Student's $t$ tests.

Immunohistochemistry. Chicks were transcardially perfused with warm $\left(\sim 40^{\circ} \mathrm{C}\right)$ PBS solution $(0.1 \mathrm{M})$, pH 7.4, followed by ice-cold $1 \%$ formaldehyde and $2 \%$ glutaraldehyde in PBS. The brains were removed from the skulls, rinsed in PBS, and sectioned at $30 \mu \mathrm{m}$ on a vibratome. After sectioning, the tissue was washed and then incubated in $1 \%$ sodium borohydride in PBS for $30 \mathrm{~min}$ at room temperature to reduce back-

ground glutaraldehyde fluorescence. After extensive washing, the tissue was incubated in block solution consisting of $2 \%$ normal goat serum, $1 \%$ bovine serum albumin (BSA), and $0.1 \%$ saponin in PBS for $1 \mathrm{~h}$ at room temperature, followed by overnight $4^{\circ} \mathrm{C}$ coincubation with primary antibodies to glycine (rabbit antiglycine; 1:200 or 1:1000; AB139, lots 0610044052 and LV1508357; Millipore Corporation) and GABA (mouse anti-GABA; 1:10,000; mAB 3A12, lot ps2; Swant) diluted in block solution. Both antibodies were raised to their respective target neurotransmitter conjugated to BSA by glutaraldehyde and have been used previously in chick nervous tissue (Matute and Streit, 1986; Kalloniatis and Fletcher, 1993). After primary antibody incubation, sections were washed and incubated for $2 \mathrm{~h}$ at room temperature with fluorescence-conjugated secondary antibodies (Alexa Fluor 488 goat anti-mouse and Alexa Fluor 568 or 633 goat anti-rabbit; 1:500; Invitrogen). After washing, sections were mounted on slides, dehydrated in ascending alcohols, and delipidized in xylenes. The tissue was then rehydrated and coverslipped using Fluoromount G medium (Southern Biotechnology Associates).

Antibody-specificity controls. Preadsorption control experiments were performed to confirm specificity of the anti-Gly and anti-GABA antibodies under the fixation and tissue preparation conditions used in this study (supplemental Fig. 1, available at www.jneurosci.org as supplemental material). Anti-Gly or antiGABA antibodies were preincubated with glycine-BSA or GABA-BSA conjugates (Abcam) for $24-48 \mathrm{~h}$ at $4^{\circ} \mathrm{C}$ before application to tissue sections. Preadsorption control tissue was compared in parallel with tissue from the same animal in which the antibodies were applied without preadsorption. Preincubation of antiGly antibody with a 100 -fold molar excess of Gly-BSA protein eliminated glycine-like immunoreactivity (supplemental Fig. $1 A, B$, available at www.jneurosci.org as supplemental material). Likewise, preincubation of anti-GABA antibody with a 100-fold molar excess of GABA-BSA protein resulted in a greatly reduced GABA-like immunoreaction (supplemental Fig. $1 E, F$, available at www.jneurosci.org as supplemental material). When anti-Gly was preincubated with the same amount of GABA-BSA used to block the anti-GABA reaction, glycine-like labeling was preserved (supplemental Fig. 1C,D, available at www.jneurosci.org as supplemental material), and preincubation of anti-GABA with the same amount of Gly-BSA used to block anti-Gly labeling did not inhibit antiGABA labeling (supplemental Fig. $1 G, H$, available at www.jneurosci.org as supplemental material). Background fluorescence was assessed in all immunohistochemistry experiments by examining tissue processed identically to experimental samples but in which primary antibody was omitted.

Image acquisition and analysis. Fluorescence images were acquired using a confocal microscope (Olympus FV1000) by sequential scanning of Alexa Fluor 488 and Alexa Fluor 568 or Alexa Fluor 633 signals using an oil-immersion objective $(60 \times$ magnification, numerical aperture 1.42). Lack of crosstalk between fluorescence channels was confirmed by examining tissue labeled with only one of each of the secondary antibodies. Image analysis was conducted using NIH ImageJ software. Overlap be- 
tween anti-Gly and anti-GABA signals was quantified in single confocal sections acquired at a depth of $\sim 1.5-2 \mu \mathrm{m}$ below the surface of the tissue from regions within each brainstem nucleus using methods similar to those previously used by Muller et al. (2006). After applying a median filter to reduce noise, images from the different fluorescence channels were separately thresholded by eye. A binary mask was then applied to each thresholded image, and overlapping regions between the binary images representing the different immunofluorescent signals were identified. The total area of overlap for each analyzed image was determined and expressed as a percentage of the total area of anti-GABA or anti-Gly signals.

\section{Results}

\section{Evoked IPSCs exhibit region-specific decay kinetics}

To compare inhibitory synaptic transmission between NM, NL, and NA, we obtained whole-cell voltage-clamp recordings from visually identified neurons within each region in tissue slices of auditory brainstem. IPSCs were evoked by extracellular stimulation and measured in the presence of ionotropic glutamate receptor antagonists (20 $\mu \mathrm{M}$ DNQX, $100 \mu \mathrm{M}$ DL-APV).

The durations of stimulus-evoked IPSCs were distinct between the different nuclei. Although rise times were similar for IPSCs recorded in NM, NL, and NA [Table 1, only significant difference between NM and NL $(p=0.05)]$, the decay time courses were distinct among the different nuclei. IPSCs recorded in NM decayed approximately threefold slower than those in NL (Fig. 1, Table 1). In NA, IPSCs typically had a fast time course, similar to those recorded in NL. However, slower currents with kinetics resembling NM IPSCs were also observed (Fig. 1, Table 1). The decay phases of NM IPSCs were best fit by the sum of two $(n=$ 4 ) or three $(n=7)$ exponentials (see Materials and Methods). Weighted time constants were not significantly different between biexponential and three-component exponential fits $(p=0.12)$. The mean weighted time constant for IPSCs recorded in NM neurons was $26.0 \pm 5.0 \mathrm{~ms}$. NL IPSC decays were best fit by the sum of two exponentials with a weighted time constant of $8.1 \pm 2.1 \mathrm{~ms}$. Fast NA IPSCs were similar in time course to NL IPSCs, with a weighted decay time constant of $11.6 \pm 9.0 \mathrm{~ms}$. Slow NA IPSCs ( 3 of 15 cells) were best fit by the sum of three exponentials and had a weighted time constant $23.1 \pm 5.6 \mathrm{~ms}$. Identical IPSC time courses were also observed in tissue from hatchling chicks [postnatal day 0 (P0)]. In hatchlings, the mean weighted decay time constants for IPSCs were $25.6 \pm 6.2 \mathrm{~ms}$ in NM $(n=4), 8.8 \pm 2.8 \mathrm{~ms}$ in $\mathrm{NL}(n=4)$, and $12.0 \pm 5.7 \mathrm{~ms}$ in NA $(n=5)$. Although maximal IPSC peak amplitudes varied widely within each nucleus, IPSCs in NA were on average smaller than those in NM and NL (Table 1, $p<0.01$ for NA compared with NM or NL), similar to previous observations of smaller excitatory currents in NA compared with NM and NL (MacLeod and Carr, 2005; MacLeod et al., 2007).

NA is a heterogeneous nucleus composed of several types of neurons with distinct intrinsic firing characteristics (Soares et al., 2002; Fukui and Ohmori, 2003). We therefore recorded from some NA neurons using a $\mathrm{K}^{+}$-based pipette solution so that NA neurons could be characterized in current-clamp configuration before recording IPSCs. We divided neurons into two categories: those that fired only one or two spikes at the beginning of depolarizing current injection (hereafter termed "onset spiking") and those that fired multiple action potentials over the duration of positive current injection ("multiple spiking"). Previous work has subdivided repetitively firing NA neurons into several categories (Soares et al., 2002), but we grouped all non-onset firing cells together as multiple spiking neurons because we observed they could exhibit different firing patterns depending on their initial membrane potential. Furthermore, Fukui and Ohmori (2003) found that NA neurons could be classified as either tonically firing or onset firing neurons in tissue from post-hatch chicks. Evoked IPSCs recorded from onset firing or multiple spiking NA neurons had different time courses (Fig. 2). On aver- 
A

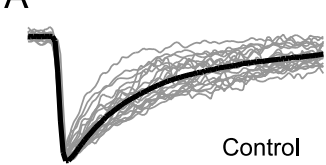

B

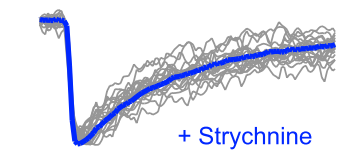

C

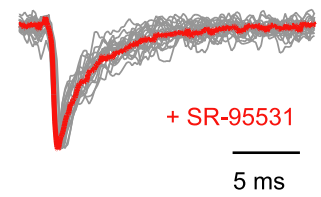

D

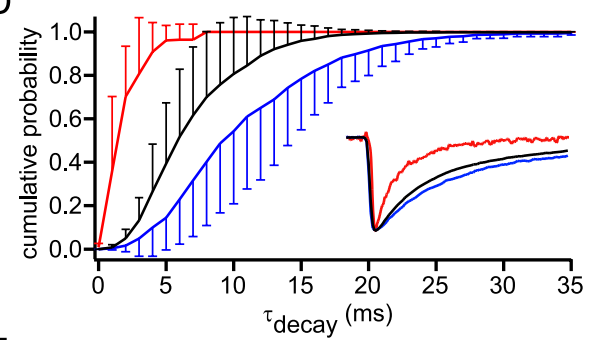

$\mathrm{E}$

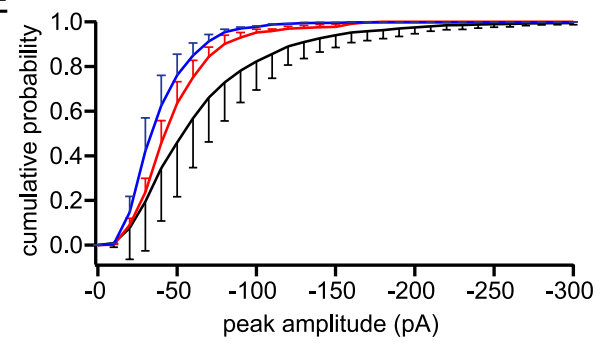

Figure 4. Spontaneous IPSC in NA indicate that GABA and glycine are coreleased. $\boldsymbol{A}-\boldsymbol{C}$, Peak-normalized average spontaneous IPSC (thick colored line) and 20 individual spontaneous IPSCs (gray lines) recorded in control conditions $(\boldsymbol{A})$ and in the presence of strychnine ( $\boldsymbol{B}$ ) or SR-95531 (C) from the same NA neuron (average IPSC from 540 events in $\boldsymbol{A}, 150$ events in $\boldsymbol{B}$, and 56 events in $\boldsymbol{C}$ ). D, Cumulative histograms of IPSC decay times for sIPSCS recorded in control (black), strychnine (red), and SR-95531 (blue). Mean \pm SD of distributions for each experimental condition from four NA cells are shown. Inset shows peak-scaled average sIPSCS under the different conditions for the single NA cell shown in A-C. sIPSC decay time distributions were significantly different between the conditions ( $p<0.0001$, Kolmogorov-Smirnov test). $\boldsymbol{E}$, Cumulative distributions of sIPSC peak amplitudes under control conditions (black) and in the presence of strychnine (red) or SR-95531 (blue) (4 cells; mean \pm SD). Peak amplitudes of events in control conditions were significantly different from those in either drug ( $p<0.0001$, Kolmogorov-Smirnov test).

age, onset spiking neurons had IPSCs that were significantly faster than those in multiple spiking neurons (weighted $\tau_{\text {decay, }}$ $2.7 \pm 0.8$ and $16.7 \pm 8.9 \mathrm{~ms}$, respectively; $p<0.001$ ). The population of multiple spiking neurons exhibited a range of IPSC decay times (weighted $\tau_{\text {decay }}$ ranged from 7.6 to $28.6 \mathrm{~ms}$ ). Although the IPSC decay values cannot be directly compared between cells recorded using the CsCl-based internal solution and the $\mathrm{K}^{+}$- based internal because of the different pipette solutions and holding potentials used $(-35.9 \mathrm{mV}$ for $\mathrm{CsCl}$ experiments, $-71.4 \mathrm{mV}$ for $\mathrm{K}^{+}$-gluconate recordings), it is likely that the slow IPSCs measured using the CsCl-based internal solution were recorded from multiple spiking neurons, whereas the fast IPSCs were recorded from both onset and multiple spiking neurons.

\section{Distinct pharmacology between the timing and intensity pathways}

We tested the neurotransmitter phenotype of evoked IPSCs by bath application of SR-95531 $(10-20 \mu \mathrm{M})$ and strychnine $(0.5$ $\mu \mathrm{M})$, which are antagonists of $\mathrm{GABA}_{\mathrm{A}}$ and glycine receptors, respectively. For IPSCs recorded in NA, we unexpectedly observed that SR-95531 application only eliminated a fraction of the inhibitory current recorded in every NA neuron tested $(n=12)$. Figure $3 A$ shows an example cell in which IPSCs were evoked under control conditions (black trace), then in the presence of SR95531 (red), then subsequently during application of strychnine after washout of SR-95531 (blue), and finally in the presence of both SR-95531 and strychnine (purple). Neither SR-95531 nor strychnine application alone completely blocked evoked currents, but both drugs together eliminated IPSCs, demonstrating the existence of GABAergic and glycinergic components of the inhibitory currents. In addition to its well characterized effect on glycine receptors, strychnine antagonizes some nicotinic acetylcholine receptor subtypes at the concentrations used in this study (Matsubayashi et al., 1998). To eliminate the possibility that the strychnine-sensitive current in NA neurons was mediated by nicotinic receptors, we performed some recordings using a low $\left[\mathrm{Cl}^{-}\right]$internal solution [CsMeSO ${ }_{3}$-based solution (see Materials and Methods); predicted $E_{\mathrm{Cl}}-=-82$ $\mathrm{mV}]$. Outward currents were recorded at a holding potential of $-30 \mathrm{mV}$ in both the presence and absence of SR-95531 under these conditions (data not shown; $n=4$ ), consistent with both components of the evoked current being mediated by $\mathrm{Cl}^{-}$-conducting channels.

As summarized in Figure 3B, glycinergic and GABAergic components of IPSCs recorded in NA neurons had distinct kinetics. The decay phases of the glycinergic component of evoked IPSCs, isolated by recording in the presence of SR-95531, were best fit by the sum of two exponentials $\left(\tau_{1}=1.8 \pm 0.4 \mathrm{~ms} ; \tau_{2}=5.0 \pm 0.7 \mathrm{~ms}\right.$; $\% \tau_{1}=41.7 \pm 3.4 \%$ ), except for two cells whose decays were fit adequately with a single-exponential function $(\tau=2.1$ and $3.8 \mathrm{~ms}$; mean $\tau_{\text {decay }}$ for all cells $=3.1 \pm 0.6$ $\mathrm{ms})$. The GABAergic component of evoked IPSCs, isolated pharmacologically using strychnine or by digital subtraction of traces acquired during SR-95531 application from control current traces, had decays best fit with the sum of two exponentials ( $\tau_{1}=6.0 \pm 1.4 \mathrm{~ms} ; \tau_{2}=35.1 \pm 4.2 \mathrm{~ms} ; \% \tau_{1}=$ $63.5 \pm 8.6 \%$ ), with a weighted time constant of $16.9 \pm 3.8 \mathrm{~ms}$.

In agreement with previous findings, evoked IPSCs in NM and NL were blocked $\geq 95 \%$ during bath application of SR-95531 $(10-20 \mu \mathrm{M})$ (Fig. 3C) (Funabiki et al., 1998; Yang et al., 1999; Lu and Trussell, 2000; Monsivais et al., 2000; Lu et al., 2005; Howard et al., 2007). In the five NA neurons recorded with the CsCl-based pipette solution, SR-95531 reduced the peak amplitude of the control IPSC by $47.6 \pm 14.2 \%$ (range of 33.6-68.4\%) (Fig. 3C).

\section{Evidence for corelease of GABA and glycine in NA}

In NA, the glycinergic and GABAergic components of evoked IPSCs that we observed could arise from the simultaneous recruitment of distinct inhibitory fibers or from corelease of both neurotransmitters from the same presynaptic neuron (Jonas et al., 1998; Awatramani et al., 2005). To distinguish between these possibilities, we recorded sIPSCs from NA neurons under control conditions and in the presence of SR-95531 or strychnine. Because these sIPSCs represented a mixture of random vesicle fusion events (miniature IPSCs) and spontaneous action potential evoked release, this approach allowed us to monitor activity of single presynaptic fibers and therefore address whether GABA and glycine can be released from the same presynaptic neuron. Figure $4 A-C$ shows example peak amplitude-scaled control sIPSCs and those recorded during SR-95531 or strychnine application for one NA neuron. A summary of IPSC decay kinetics for each condition from four NA neurons is presented in Figure $4 D$. In control conditions, the mean decay time constants for sIPSCs ranged from 1.0 to $40.2 \mathrm{~ms}$, with a mean time constant of $7.8 \pm$ $2.9 \mathrm{~ms}$ (Fig. 4A,D). Glycinergic sIPSCs recorded in the presence of SR-95531 had fast decay kinetics (mean $\tau_{\text {decay }}=2.9 \pm 1.2 \mathrm{~ms}$; range of $0.9-8.9 \mathrm{~ms}$ ) (Fig. $4 B, D$ ). The duration of GABAergic sIPSCs isolated by strychnine application exhibited a range of 

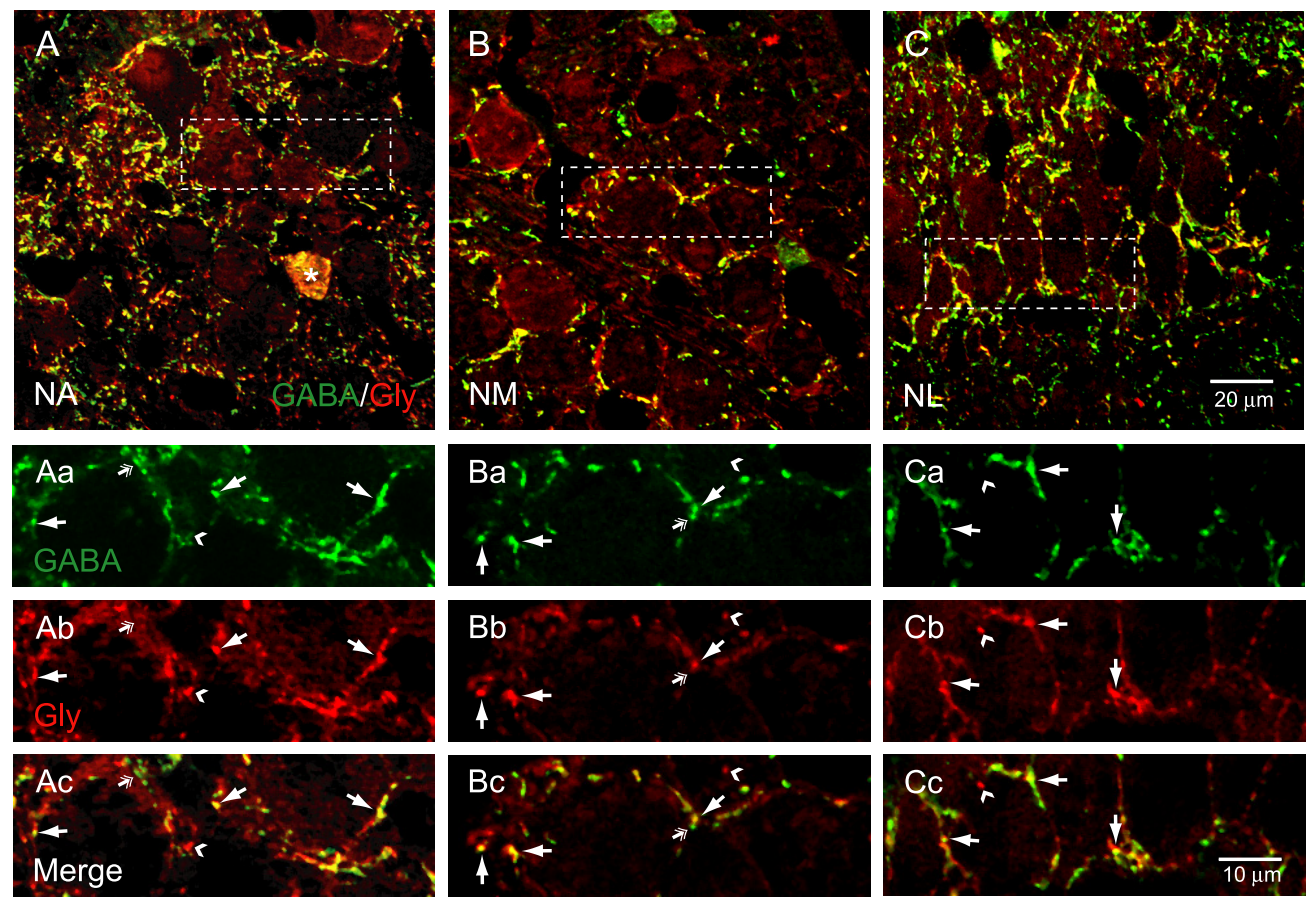

Figure 5. Colocalization of GABA and glycine in embryonic chick auditory brainstem. Immunofluorescent labeling of GABA (green) and glycine (red) in NA ( $(\boldsymbol{A})$, NM (B), and NL $(\boldsymbol{C})$ of an E18 chick. $\boldsymbol{A}-\boldsymbol{C}$, Merged stacks of confocal images acquired in $0.5 \mu \mathrm{m}$ intervals in the $z$-axis ( $\boldsymbol{A}, 18$ sections; $\boldsymbol{B}, 5$ sections; $\boldsymbol{C}, 5$ sections). $\boldsymbol{A} \boldsymbol{a}$ - $\boldsymbol{A c}$, Single confocal sections corresponding to dashed box in $\boldsymbol{A}$ showing GABA $(\boldsymbol{A a})$, glycine $(\boldsymbol{A} \boldsymbol{b})$, and both fluorescent signals merged $(\boldsymbol{A c})$. Ba-Bc and $\boldsymbol{C} \boldsymbol{a}-\boldsymbol{C}$ c show same for NM and NL, respectively. Arrows indicate double-labeled puncta. Arrowheads indicate bouton-like structures showing only glycine labeling. Double arrows show puncta with GABA labeling only. Asterisk in $\boldsymbol{A}$ denotes a cell body exhibiting labeling for GABA and glycine. Cell bodies exhibiting GABA-like and/or glycine-like immunoreactivity were infrequently observed within NA $(<3-4$ cells when present, typically no cells observed). Scale bar in $C$ applies to $A-C$. Scale bar in Cc applies to $\mathrm{Aa}-\mathrm{Cc}$.

values that were on average slower than glycinergic IPSCs (mean $\tau_{\text {decay }}=11.7 \mathrm{~ms} \pm 4.3 \mathrm{~ms}$; range of $1.6-43.6 \mathrm{~ms}$ ) (Fig. $\left.4 C, D\right)$. The broad distribution of sIPSC decay kinetics recorded in the presence of strychnine and overlap with decay kinetics of events recorded in SR-95531 prevented a quantitative assessment of the relative contributions of GABA and glycine-mediated transmission to spontaneous events. However, the significant shift in the overall population of $\tau_{\text {decay }}$ values recorded under control conditions to faster or slower values in the presence of SR-95531 or strychnine, respectively (Fig. $4 D)(p<0.0001$, KolmogorovSmirnov test), indicates that a considerable number of control sIPSCs arose from the release of both GABA and glycine from the same axon. If most IPSCs arose from release of GABA only or glycine only, under control conditions, we should have observed two separate populations of decays with means matching those recorded in SR-95531 or strychnine, which would have appeared as a biphasic curve in the cumulative probability plot. The observation that SR-95531 or strychnine application significantly reduced the amplitudes of sIPSCs (Fig. $4 E)(p<0.0001$, Kolmogorov-Smirnov test) also supports this interpretation.

As a complementary approach for investigating whether GABA and glycine arise from the same or different sources, we examined the expression patterns of these neurotransmitters in the chick auditory brainstem using immunohistochemistry. Double-immunofluorescent labeling with a GABA-specific antibody in combination with a glycine-specific antibody revealed punctate expression of both neurotransmitters around cell bodies and in the neuropil of NA (Fig. $5 A$ ). These puncta likely correspond to inhibitory nerve terminals contacting NA neurons. Bouton-like structures exhibiting only GABA-like immunoreactivity or only glycine-like immunoreactivity were observed (Fig. $5 A$, double arrows and arrowheads, respectively), but many puncta also exhibited colabeling for both neurotransmitters (Fig $5 A$, arrows). In NA, $36.4 \pm 5.2 \%$ of the glycine-like signal also exhibited GABA-like immunoreactivity (\%GABA/Gly), whereas $36.8 \pm 10.1 \%$ of GABA-like labeling also had glycine-like immunoreactivity (\%Gly/GABA). Interestingly, glycine labeling was also observed in NM and NL (Fig. 5B,C), despite the apparent lack of glycine receptor-mediated currents in these nuclei (Fig. $3 C$ ). Similar overlap between GABA and glycine signals was observed in NM ( $42.3 \pm 16.6 \% \%$ GABA/Gly; $47.5 \pm 6.9 \% \%$ Gly/ GABA) and NL (36.6 $\pm 12.9 \%$ \%GABA/Gly; $33.9 \pm 4.1 \%$ \% Gly/ GABA) ( $p>0.05$ for all comparisons between nuclei; data from three different chicks, one image/brainstem region analyzed per animal). Together with our sIPSC recordings, these findings suggest that GABA and glycine can be coreleased from the same presynaptic axon in NA and possibly also in NM and NL.

\section{Depolarizing $E_{\text {gly }}$ in NA}

A unique feature of inhibition in NM is that GABAergic currents have a depolarizing effect on NM neurons throughout development (Lu and Trussell, 2001; Monsivais and Rubel, 2001; Howard et al., 2007). GABAergic transmission remains inhibitory under most circumstances in mature animals because of high expression levels of low-threshold activated $\mathrm{K}^{+}$channels in NM neurons (Howard et al., 2007). Because the majority of NA neurons have fundamentally different intrinsic membrane properties compared with NM neurons (Soares et al., 2002; Fukui and Ohmori, 2003) and less robust low-threshold $\mathrm{K}^{+}$conductances (Fukui and Ohmori, 2003), we examined the polarity of inhibition in NA (Fig. 6). To estimate the reversal potential for $\mathrm{Cl}^{-}$mediated conductances, we measured currents in response to exogenously applied glycine using the gramicidin perforatedpatch technique (Ebihara et al., 1995). In the majority of these 
A

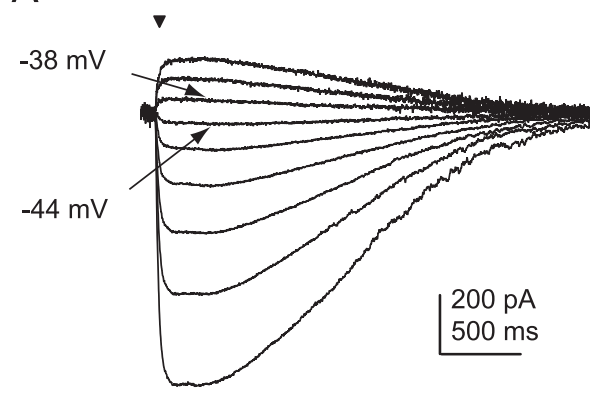

B

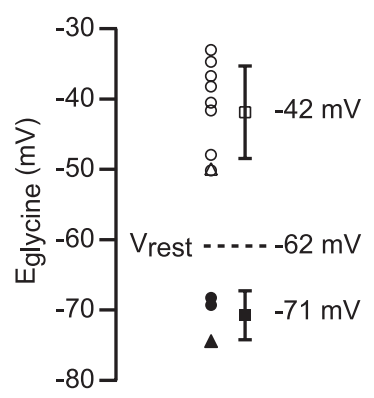

C

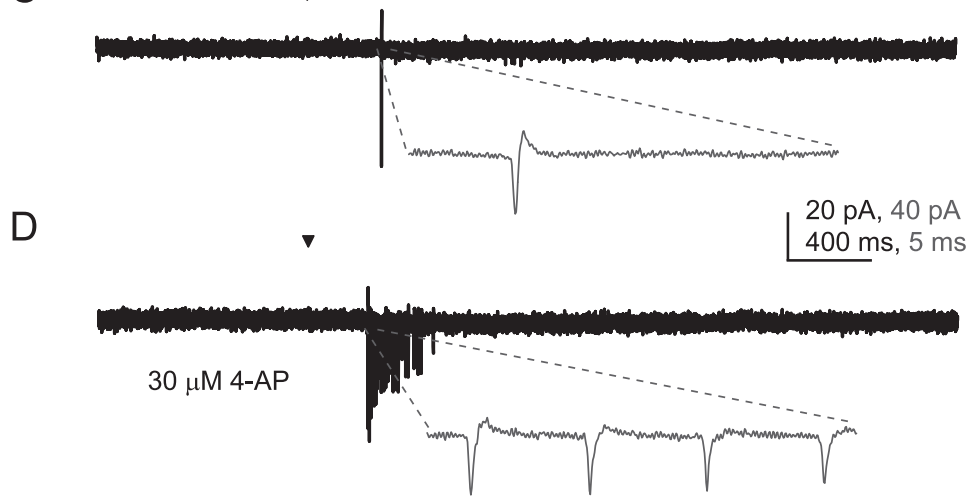

Figure 6. Depolarizing reversal potential for glycine-mediated currents in NA. $A$, Example responses recorded from an NA neuron using the gramicidin perforated-patch technique. Currents were recorded in response to a $100 \mathrm{~ms}$ puff of glycine (1 mm; inverted triangle indicates onset of application) at a series of holding potentials between -85 and $-27 \mathrm{mV}$. B, Summary of reversal potentials for glycinergic currents $\left(E_{\text {gly }}\right)$ in NA neurons. Circles show $E_{\text {gly }}$ values for multiple spiking cells. Triangles show measurements for onset spiking cells. Squares show average values for depolarizing (open) and hyperpolarizing (filled) $E_{\text {gly }}$ values. C, Cell-attached recording from an NA neuron. Glycine application elicited an action potential (see inset). D, Cell-attached recording from a different NA neuron obtained in the presence of $30 \mu \mathrm{m}$ 4-AP. Cell spiked multiple times in response to glycine exposure. In both $\boldsymbol{C}$ and $\boldsymbol{D}, 1 \mathrm{~mm}$ glycine was applied for $1 \mathrm{~s}$ starting at the time denoted by the inverted triangle.

recordings (9 of 12 cells), the reversal potential for glycineinduced currents $\left(E_{\mathrm{gly}}\right)$ was depolarized compared with resting membrane potential (mean $E_{\text {gly }}=-41.5 \pm 6.6 \mathrm{mV}$; mean resting membrane potential for all cells tested, $-61.9 \pm 3.5 \mathrm{mV}$ ). A small subset of cells ( 3 of 13) was also observed with hyperpolarized $E_{\text {gly }}$ values $(-70.8 \pm 3.5 \mathrm{mV})$. In the NA neurons we studied, $E_{\mathrm{gly}}$ values did not seem to correlate with the age of tissue (E16-E20) or the action potential firing phenotype of the recorded cells. To confirm our results from gramicidin perforated-patch recordings, we also made extracellular recordings from several NA neurons in the cell-attached configuration. In three of four neurons, puff application of $1 \mathrm{~mm}$ glycine caused the cell to fire a single action potential (see example in Fig. 6C). We also performed cell-attached recordings from four cells in the presence of the $\mathrm{K}^{+}$-channel blocker 4-aminopyridine (4-AP) $(30 \mu \mathrm{M})$, which was added to enhance excitability in our slices. Again, action potentials were recorded in response to glycine application in three of the four neurons examined. Two of these cells fired multiple action potentials in response to glycine (for example, see Fig. $6 D$ ), one cell fired a single action potential, and the last cell did not exhibit any spiking activity. Together, our results from gramicidin perforated-patch and cell-attached recordings indicate that most NA neurons in late embryonic chick tissue have depolarized $\mathrm{Cl}^{-}$reversal potentials. Cell-attached recordings were additionally used to examine responses to glycine application in NA neurons in tissue from hatchling chicks (P0). In hatchling tissue, four cells responded to glycine puffs with action potential firing (either from rest or increased spiking above spontaneous firing), whereas three cells with spontaneous spiking activity responded with a decrease in spike output. Ten additional cells tested did not exhibit changes in action potential firing with glycine application.

\section{Excitatory effects of GABA/glycine in NA}

Given that the depolarizing $E_{\text {gly }}$ values measured for the majority of NA neurons studied was above action potential threshold $(-47.2 \pm 1.4 \mathrm{mV}$ in multiple spiking neurons, $-51.1 \pm 2.0 \mathrm{mV}$ onset spiking cells), we examined whether GABAergic/ glycinergic inputs to NA neurons could exert an excitatory effect on these cells. To approximate the depolarizing $E_{\text {gly }}$ values we measured using gramicidin perforated-patch recordings, whole-cell recordings were acquired using a $\mathrm{K}^{+}$-based internal solution with a $\left[\mathrm{Cl}^{-}\right]$calculated to yield

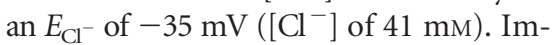
mediately after acquiring whole-cell recordings, cellular firing characteristics of NA neurons were assessed by current injection in current-clamp mode. After measuring IPSCs in response to repetitive stimulation in voltage clamp, recordings were switched back to current-clamp and responses with the same stimulus pattern was investigated. Figure 7 shows example responses from an onset spiking cell (Fig. $7 A-C$ ) and a multiple spiking neuron (Fig. $7 D-F$ ). In both cell types, postsynaptic potentials recorded in the presence of glutamate receptor blockers could elicit action potentials. Of four onset neurons tested, three fired at least one action potential over the course of 10 stimuli applied at $100 \mathrm{~Hz}$. Similarly, four of five multiple spiking neurons fired action potentials in response to stimulation of inhibitory fibers.

\section{Discussion}

We found that the time course and neurotransmitter phenotype of inhibitory currents were distinct between NM, NL, and NA. These divergent properties may contribute to the unique computational tasks performed in the different brainstem regions. Additionally, we observed that glycinergic/GABAergic inputs could excite NA neurons, which could have important implications for the recruitment of SON activity.

\section{Implications for ITD coding}

The approximately threefold difference in IPSC decay kinetics we measured between NM and NL neurons indicates that inhibition may have a more complex role in ITD processing than appreciated previously. Most proposals for how inhibition contributes to ITD coding assume a slow time course for GABAergic currents (Funabiki et al., 1998; Monsivais et al., 2000; Grothe, 2003; Burger et al., 2005; Dasika et al., 2005). In NM, it is clear that inhibitory inputs do not preserve temporal information but instead exert a tonic influence (Lu and Trussell, 2000). Our results raise the possibility that inhibition may have more phasic effects in NL compared with NM. How- 
ever, timing information is probably not retained in the activity of GABAergic inputs to NL. SON neurons do not phase lock as well as NM cells (Lachica et al., 1994) and do not appear to express intrinsic properties suited to preservation of timing information (Yang et al., 1999). However, heterogeneity in both tone-evoked response properties and anatomical features has been observed in SON neurons (Carr et al., 1989; Lachica et al., 1994). It is possible that a subpopulation of SON neurons may provide phase-locked inhibitory signals to NL, although no evidence exists to suggest that this may be the case. Further work will be needed to resolve whether precisely timed inhibition contributes to NL function.

Previous studies did not observe fast kinetics for inhibition in NL neurons from similarly aged chicks when recordings were performed at room temperature (Funabiki et al., 1998; Yang et al., 1999). Because our recordings were obtained closer to physiological temperature $\left(41^{\circ} \mathrm{C}\right.$ in chickens), the measurements presented here likely more closely resemble in vivo inhibitory currents than those reported previously.

\section{Glycinergic transmission in the avian auditory brainstem}

Perhaps the most surprising finding of this study was that glycinergic transmission contributes significantly to nonglutamatergic currents in all NA neurons examined. Glycinergic transmission had not been believed previously to play an important role in the bird auditory system because of a lack of immunohistochemical evidence for significant glycine expression in the chicken auditory nuclei (Code and Rubel, 1989) as well as observations that inhibition in NM and NL could be completely abolished by antagonists of $\mathrm{GABA}_{\mathrm{A}}$ receptors (Funabiki et al., 1998; Lu and Trussell, 2000). Our pharmacological and immunohistochemical evidence for glycinergic transmission raise the possibility that, at least within NA, inhibition in the avian and mammalian auditory systems is more similar than originally believed. Because our study focused on late-stage embryos, it is possible that the glycinergic currents we observed are not a permanent feature but instead reflect a transient stage of NA development. However, glycine-like immunoreactivity remains abundant in the NA of hatchling chicks $(\mathrm{P} 0)$, and hatchling NA neurons still respond to glycine application (data not shown). Thus, glycinergic transmission likely still persists at an age at which NA neurons exhibit well developed intrinsic properties (Fukui and Ohmori, 2003) and hearing is intact.

Interestingly, immunofluorescent labeling also revealed coexpression of GABA and glycine in NM and NL, despite the lack of glycinergic synaptic currents in these regions. Whether glycine release within NM and NL has any function in these nuclei despite the apparent lack of postsynaptic glycine receptors remains to be determined. Glycine could potentially modulate excitatory and/or inhibitory transmission by acting on presynaptic glycine receptors (Turecek and Trussell, 2001) or extrasynaptic receptors on NM or NL neurons. Coreleased GABA was demonstrated recently to speed the decay of glycinergic currents by acting as a coagonist at glycine receptors ( $\mathrm{Lu}$ et al., 2008). An intriguing possibility is that coreleased glycine could act to modulate the response of postsynaptic GABA receptors on NL and NM neurons.

\section{Depolarizing $E_{\mathrm{Cl}^{-}}$in NA}

An important caveat to our estimation of $\mathrm{Cl}^{-}$reversal potential is that our experiments were performed in late-stage chick embryos. Although we did not observe any obvious relationship between tissue age and $E_{\mathrm{Cl}^{-}}$in the cells we tested, we cannot rule out the possibility that our experiments were conducted before a developmental switch in the polarity of inhibition in NA. Using cell-attached recordings, we found that NA neurons in tissue from hatchling chicks could fire action potentials in response to glycine application, demonstrating that NA neurons in more mature tissue can also have a depolarized $E_{\mathrm{Cl}^{-}}$value. However, inhibitory responses were also observed in some cells, and most cells did not respond to glycine puffs with spiking activity, although the lack of responses in these latter cells could be attributable to reduced excitability, perhaps as a result of higher lowthreshold $\mathrm{K}^{+}$channel expression (Fukui and Ohmori, 2003). Importantly, in vivo recordings have demonstrated clear inhibitory influences in the sound-evoked response properties of mature NA units (Warchol and Dallos, 1990; Koppl and Carr, 2003). The obvious inhibition of spiking activity observed in some NA cells in response to presentation of tone or noise stimuli may indicate that GABAergic/glycinergic inputs are hyperpolarizing in at least some cell types in the mature animal.

Our current-clamp experiments indicate that GABAergic/glycinergic postsynaptic potentials may exert an excitatory influence on NA neurons under certain conditions. It should be noted that we observed a range of depolarizing $E_{\text {gly }}$ values, as well some instances in which $E_{\mathrm{gly}}$ was hyperpolarizing. Differential expression or activity of $\mathrm{Na}^{+} / \mathrm{K}^{+} / \mathrm{Cl}^{-}$transporters and/or $\mathrm{K}^{+} / \mathrm{Cl}^{-}$ transporters could underlie differences in $E_{\mathrm{Cl}^{-}}$(Payne et al., 2003). If SON neurons project to the same NA neurons from which they receive input, excitatory GABA/glycine influences in 
NA could establish a positive feedback loop that would increase activity in both nuclei. This could in turn alter the level of inhibition to NM and NL neurons and thereby enhance the functional range of inhibitory signaling in the brainstem. Positive feedback could also enhance the range of sound intensity coding by NA neurons by amplifying changes in the level of auditory nerve input to NA. In this scenario, additional mechanisms, such as synaptic depression, spike threshold accommodation, or metabotropic receptor activity (e.g., $\mathrm{GABA}_{\mathrm{B}}$ receptors), would be required to prevent overexcitability.

\section{Sources of GABAergic/glycinergic input}

Several anatomical studies have identified robust projections from ipsilateral SON to NM, NL, and NA (Lachica et al., 1994; Yang et al., 1999; Burger et al., 2005; Nishino et al., 2008), which has led to the prevailing view that the majority of GABAergic input to the timing and intensity pathways arises from SON. In partial support of this view, Nishino et al. (2008) recently demonstrated that lesioning SON altered ITD coding in the ipsilateral NL of chickens, presumably as a result of a loss of inhibitory input to NM and NL. Because our electrophysiological and immunohistochemical data indicate that a significant amount of GABAergic and glycinergic innervation arises from a common source, we consider it likely that SON can provide mixed GABAergic/glycinergic input to the different brainstem nuclei. We occasionally observed a few GABA and/or glycine-like immunoreactive cell bodies within or around NA (Fig. 5A) and in neurons between NM and NL (data not shown), consistent with previous reports of GABAergic neurons adjacent to or within these nuclei (Müller, 1987; Carr et al., 1989; von Bartheld et al., 1989). Thus, non-SON sources may also contribute some GABAergic and/or glycinergic input to NM, NL, and NA, although this may represent a small fraction of the input to these nuclei.

Although we did not investigate the specific mechanisms underlying the unique features of GABAergic and glycinergic currents in NM, NL, and NA, it is likely that postsynaptic factors had an important role. Differences in postsynaptic $\mathrm{GABA}_{\mathrm{A}}$ receptor subunit composition or receptor modulation probably accounts for the distinct decay kinetics between NM and NL. Differences in release time course are not likely to contribute to the observed differences in IPSC kinetics because, in NM, IPSCs evoked at the low stimulus frequencies used in this study have identical kinetics to miniature IPSCs, indicating that evoked release is highly synchronous in these cells at low stimulus frequency ( $\mathrm{Lu}$ and Trussell, 2000). Because glycine appears to be expressed presynaptically not only in NA but also in NM and NL, the presence or absence of postsynaptic glycine receptors likely underlies the pharmacological differences between the sound intensity and timing pathways, similar to previous findings of postsynaptic selection of coreleased GABA and glycine in the mammalian cerebellum (Dugué et al., 2005). Considering that most of the inhibitory input to NM, NL, and NA likely arises from ipsilateral SON, the divergent properties of inhibitory transmission that we observed suggest that postsynaptic specializations permit segregation of inhibitory influences between different functional pathways despite a common source.

\section{References}

Awatramani GB, Turecek R, Trussell LO (2004) Inhibitory control at a synaptic relay. J Neurosci 24:2643-2647.

Awatramani GB, Turecek R, Trussell LO (2005) Staggered development of GABAergic and glycinergic transmission in the MNTB. J Neurophysiol 93:819-828.
Boord RL (1968) Ascending projections of the primary cochlear nuclei and nucleus laminaris in the pigeon. J Comp Neurol 133:523-541.

Burger RM, Cramer KS, Pfeiffer JD, Rubel EW (2005) Avian superior olivary nucleus provides divergent inhibitory input to parallel auditory pathways. J Comp Neurol 481:6-18.

Carr CE, Konishi M (1990) A circuit for detection of interaural time differences in the brain stem of the barn owl. J Neurosci 10:3227-3246.

Carr CE, Soares D (2002) Evolutionary convergence and shared computational principles in the auditory system. Brain Behav Evol 59:294-311.

Carr CE, Fujita I, Konishi M (1989) Distribution of GABAergic neurons and terminals in the auditory system of the barn owl. J Comp Neurol 286:190-207.

Code RA, Rubel EW (1989) Glycine-immunoreactivity in the auditory brain stem of the chick. Hear Res 40:167-172.

Dasika VK, White JA, Carney LH, Colburn HS (2005) Effects of inhibitory feedback in a network model of avian brain stem. J Neurophysiol 94:400-414.

Dugué GP, Dumoulin A, Triller A, Dieudonné S (2005) Target-dependent use of co-released inhibitory transmitters at central synapses. J Neurosci 25:6490-6498.

Ebihara S, Shirato K, Harata N, Akaike N (1995) Gramicidin-perforated patch recording: GABA response in mammalian neurones with intact intracellular chloride. J Physiol 484:77-86.

Fukui I, Ohmori H (2003) Developmental changes in membrane excitability and morphology of neurons in the nucleus angularis of the chicken. J Physiol 548:219-232.

Fukui I, Sato T, Ohmori H (2006) Improvement of phase information at low sound frequency in nucleus magnocellularis of the chicken. J Neurophysiol 96:633-641.

Funabiki K, Koyano K, Ohmori H (1998) The role of GABAergic inputs for coincidence detection in the neurones of nucleus laminaris of the chick. J Physiol 508:851-869.

Grothe B (2003) New roles for synaptic inhibition in sound localization. Nat Rev Neurosci 4:540-550.

Howard MA, Burger RM, Rubel EW (2007) A developmental switch to GABAergic inhibition dependent on increases in Kv1-type $\mathrm{K}^{+}$currents. J Neurosci 27:2112-2123.

Jonas P, Bischofberger J, Sandkühler J (1998) Corelease of two fast neurotransmitters at a central synapse. Science 281:419-424.

Kalloniatis M, Fletcher EL (1993) Immunocytochemical localization of the amino acid neurotransmitters in the chicken retina. J Comp Neurol 336:174-193.

Kim Y, Trussell LO (2007) Ion channels generating complex spikes in cartwheel cells of the dorsal cochlear nucleus. J Neurophysiol 97:1705-1725.

Konishi M (2003) Coding of auditory space. Annu Rev Neurosci 26:31-55.

Köppl C, Carr CE (2003) Computational diversity in the cochlear nucleus angularis of the barn owl. J Neurophysiol 89:2313-2329.

Kotak VC, Korada S, Schwartz IR, Sanes DH (1998) A developmental shift from GABAergic to glycinergic transmission in the central auditory system. J Neurosci 18:4646-4655.

Lachica EA, Rübsamen R, Rubel EW (1994) GABAergic terminals in nucleus magnocellularis and laminaris originate from the superior olivary nucleus. J Comp Neurol 348:403-418.

Lu T, Trussell LO (2000) Inhibitory transmission mediated by asynchronous transmitter release. Neuron 26:683-694.

Lu T, Trussell LO (2001) Mixed excitatory and inhibitory GABA-mediated transmission in chick cochlear nucleus. J Physiol 535:125-131.

Lu T, Rubio ME, Trussell LO (2008) Glycinergic transmission shaped by the corelease of GABA in a mammalian auditory synapse. Neuron 57:524-535.

Lu Y, Burger RM, Rubel EW (2005) GABA(B) receptor activation modulates $\mathrm{GABA}(\mathrm{A})$ receptor-mediated inhibition in chicken nucleus magnocellularis neurons. J Neurophysiol 93:1429-1438.

MacLeod KM, Carr CE (2005) Synaptic physiology in the cochlear nucleus angularis of the chick. J Neurophysiol 93:2520-2529.

MacLeod KM, Carr CE (2007) Beyond timing in the auditory brainstem: intensity coding in the avian cochlear nucleus angularis. Prog Brain Res 165:123-133.

MacLeod KM, Horiuchi TK, Carr CE (2007) A role for short-term synaptic facilitation and depression in the processing of intensity information in the auditory brain stem. J Neurophysiol 97:2863-2874.

Magnusson AK, Kapfer C, Grothe B, Koch U (2005) Maturation of glycin- 
ergic inhibition in the gerbil medial superior olive after hearing onset. J Physiol 568:497-512.

Matsubayashi H, Alkondon M, Pereira EF, Swanson KL, Albuquerque EX (1998) Strychnine: a potent competitive antagonist of alpha-bungarotoxinsensitive nicotinic acetylcholine receptors in rat hippocampal neurons. J Pharmacol Exp Ther 284:904-913.

Matute C, Streit P (1986) Monoclonal antibodies demonstrating GABA-like immunoreactivity. Histochemistry 86:147-157.

Monsivais P, Rubel EW (2001) Accommodation enhances depolarizing inhibition in central neurons. J Neurosci 21:7823-7830.

Monsivais P, Yang L, Rubel EW (2000) GABAergic inhibition in nucleus magnocellularis: implications for phase locking in the avian auditory brainstem. J Neurosci 20:2954-2963.

Müller CM (1987) gamma-Aminobutyric acid immunoreactivity in brainstem auditory nuclei of the chicken. Neurosci Lett 77:272-276.

Muller E, Le Corronc H, Triller A, Legendre P (2006) Developmental dissociation of presynaptic inhibitory neurotransmitter and postsynaptic receptor clustering in the hypoglossal nucleus. Mol Cell Neurosci 32:254-273.

Nabekura J, Katsurabayashi S, Kakazu Y, Shibata S, Matsubara A, Jinno S, Mizoguchi Y, Sasaki A, Ishibashi H (2004) Developmental switch from GABA to glycine release in single central synaptic terminals. Nat Neurosci 7:17-23.

Nishino E, Yamada R, Kuba H, Hioki H, Furuta T, Kaneko T, Ohmori H (2008) Sound-intensity-dependent compensation for the small interaural time difference cue for sound source localization. J Neurosci 28:71537164 .

Oertel D (1999) The role of timing in the brain stem auditory nuclei of vertebrates. Annu Rev Physiol 61:497-519.

Parks TN, Rubel EW (1975) Organization and development of brain stem auditory nuclei of the chicken: organization of projections from n. magnocellularis to $n$. laminaris. J Comp Neurol 164:435-448.
Parks TN, Rubel EW (1978) Organization and development of the brain stem auditory nuclei of the chicken: primary afferent projections. J Comp Neurol 180:439-448.

Payne JA, Rivera C, Voipio J, Kaila K (2003) Cation-chloride cotransporters in neuronal communication, development and trauma. Trends Neurosci 26:199-206.

Rubel EW, Parks TN (1975) Organization and development of brain stem auditory nuclei of the chicken: tonotopic organization of n. magnocellularis and n. laminaris. J Comp Neurol 164:411-433.

Smith AJ, Owens S, Forsythe ID (2000) Characterisation of inhibitory and excitatory postsynaptic currents of the rat medial superior olive. J Physiol 529:681-698.

Soares D, Chitwood RA, Hyson RL, Carr CE (2002) Intrinsic neuronal properties of the chick nucleus angularis. J Neurophysiol 88:152-162.

Sullivan WE, Konishi M (1984) Segregation of stimulus phase and intensity coding in the cochlear nucleus of the barn owl. J Neurosci 4:1787-1799.

Takahashi T, Moiseff A, Konishi M (1984) Time and intensity cues are processed independently in the auditory system of the owl. J Neurosci 4:1781-1786.

Trussell LO (1999) Synaptic mechanisms for coding timing in auditory neurons. Annu Rev Physiol 61:477-496.

Turecek R, Trussell LO (2001) Presynaptic glycine receptors enhance transmitter release at a mammalian central synapse. Nature 411:587-590.

von Bartheld CS, Code RA, Rubel EW (1989) GABAergic neurons in brainstem auditory nuclei of the chick: distribution, morphology, and connectivity. J Comp Neurol 287:470-483.

Warchol ME, Dallos P (1990) Neural coding in the chick cochlear nucleus. J Comp Physiol A 166:721-734.

Yang L, Monsivais P, Rubel EW (1999) The superior olivary nucleus and its influence on nucleus laminaris: a source of inhibitory feedback for coincidence detection in the avian auditory brainstem. J Neurosci 19:2313-2325. 